\title{
Fuzzy Clustering for Next Generation Wireless Sensor Networks
}

\author{
Ola Albeshri ${ }^{1}$, Laila Nassef ${ }^{1}$, Etimad Fadel ${ }^{1}$ \\ King Abdulaziz University, Department of Computer Science, Faculty of Computing and Information Technology, \\ Jeddah, Saudi Arabia ${ }^{1}$
}

\begin{abstract}
The massive growing demands for radio wireless communications have resulted in a spectrum scarcity problem. Cognitive radios utilize a dynamic spectrum access to share the spectrum with licensed frequency bands in an opportunistic manner. The integration of cognitive radio with wireless sensor nodes can improve spectrum utilization and increase communication quality. Currently, clustering protocols have been developed to minimize energy consumption and to prolong network's lifetime. However, spectrum awareness in not considered. Thus, clustering protocols need to adapt to the changes in the surrounding environment and to optimally consider both energy efficiency and spectrum awareness. Therefore, this paper develops a Fuzzy based Energy Efficient and Spectrum Aware clustering protocol (FEESA) to optimally elect cluster heads based on four conflicting parameters: residual energy, distance to base station, node degree, and channel availability. The performance of the proposed protocol is simulated using MATLAB and Mamdani fuzzy inference system. Two simulation scenarios and three performance metrics were used. The proposed fuzzy clustering protocol is compared with three different protocols: a basic energy efficient protocol, a spectrum aware protocol, and an energy efficient spectrum aware protocol which uses a weighting function. The simulation results indicate the effectiveness of the proposed fuzzy based clustering protocol to extend network lifetime and reduce energy consumption.
\end{abstract}

Keywords: Routing Protocols, Cognitive Radio Sensor Networks, Spectrum Aware, Fuzzy Logic.

\section{INTRODUCTION}

Wireless Sensor Networks (WSNs) have emerged as a promising technology to provide connectivity to a huge number of nodes [1]. WSNs can sense, perform computation, take decisions and transmit useful collected data to the Base Station (BS) through fixed unlicensed spectrum bands [2]. Many routing protocols have been proposed for traditional cluster based WSNs, where nodes are divided into different virtual groups according to a set of rules.

The Low Energy Adaptive Clustering Hierarchy (LEACH) protocol [3] is a basic clustering protocol. Cluster Heads (CHs) are elected by generating a number between zero and one and if it is less than a predefined threshold $\mathrm{T}(\mathrm{n})$, then this node will become the $\mathrm{CH}$ in the next round. The threshold value is defined in equation (1):

$$
T(n)= \begin{cases}\frac{p}{1-p *\left(r \bmod \frac{1}{p}\right)}, & \text { if } n \in G \\ 0, & \text { otherwise }\end{cases}
$$

Where, $P$ is the desired $\mathrm{CH}$ percentage, $r$ is current round, $G$ is the set of nodes that have not been $\mathrm{CHs}$ in 1/P rounds.

The Hybrid Energy-Efficient Distributed (HEED) clustering protocol [4] adds energy level and node distance to its neighbors to $\mathrm{CH}$ election criteria. However, both protocols use the unlicensed, fixed spectrum assignments and suffer from spectrum scarcity problem.

WSNs coexist with other interfering wireless technologies like Wi-Fi and Bluetooth which degrade performance of all communicating networks [5]. Cognitive Radio (CR) provides the key enabling functions for the next generation wireless communication [6]. It uses an intelligent radio technology that is aware of the changes of its surrounding environment and can adapt its operating parameters dynamically to solve spectrum scarcity problem. It utilizes a Dynamic Spectrum Access (DSA) [7] to sense spectrum and to share available vacant channels in licensed frequency band. The integration of CR with WSNs have resulted in a new networking paradigm called Cognitive Radio Sensor Networks (CRSNs) [8].

Routing protocols proposed for traditional WSNs have been designed to be energy efficient. They are not suitable for 
CRSN which need to consider both energy efficiency and spectrum awareness [9]. Therefore, basic clustering algorithms need some modifications to dynamically utilize licenced frequency bands and to provide spectrum awareness.

CogLEACH [10] added the channel availability as part of the $\mathrm{CH}$ selection criteria. CogLEACH-C [11] extended CogLEACH by adding nodes remaining energy to $\mathrm{CH}$ selection in a centralized architecture. The energy aware event driven (ESAC) [12] forms clusters only after an event is being detected and maintain these clusters until the end of the event. The mobile version of ESAC (mESAC) is also an event driven protocol [13] and both form clusters between event and the sink node. The energy aware Event-driven Routing Protocol (ERP) also creates routes from nodes that detect event to the sink node [14].

Gupta [15] proposed fuzzy logic based protocol to optimally elect CHs based on three fuzzy descriptors of residual energy, concentration, and centrality. His protocol improved lifetime of the network as compared to LEACH [16]. However, the main drawbacks of his protocol was the centralized election mechanism, where each node has to send information about its current location and energy level to BS in each round [17]. Anno [18] also used the fuzzy logic to elect $\mathrm{CHs}$ based on remaining energy, the distance of cluster centroid, and network traffics as inputs fuzzy parameters. His work does not suggest how the inputs descriptors are collected and how the fuzzy logic is run [19].

All of the above protocols are energy efficient and spectrum aware with no optimal selection criteria for CHs. Thus there is a need to optimally develop energy efficient and spectrum aware protocol for the CRSNs to enhance network lifetime.

Therefore, this paper develops a Fuzzy based Energy Efficient and Spectrum Aware clustering protocol (FEESA) for CRSNs that optimally elect CHs based on four proposed parameters of residual energy, distance to base station, node degree, and channel availability. Mamdani's Fuzzy Inference System (FIS) is used to take optimal decisions using membership functions and fuzzy logic operators [20]. The simulation results indicated the effectiveness of the proposed fuzzy-based clustering protocol in terms of network lifetime and energy consumption.

The rest of this paper is organized as follows: section 2 presents proposed FEESA protocol in details explanation. The performance of the FEESA protocol is evaluated in section 3. Finally, section 4 concludes results and presents future research.

\section{FUZZY-BASED ENERGY EFFICIENT SPECTRUM AWARE (FEESA) ROUTING PROTOCOL}

CRSNs consist of N CR enabled sensor nodes called Secondary Users (SUs) which coexist with M Primary Users (PUs) that operate on a set of orthogonal C frequency channels. Both SUs and PU are randomly deployed and assumed to be static. SUs can opportunistically access the spectrum when no PU is operating on licensed frequency band. The number of available channels can be identified by their unique channel IDs. Each node is assumed to have a list of all available spectrum bands depending on its location. Also SUs can estimate the PU appearance probability and PU idle time in the licensed channel in a distributed way. This information will be used to determine vacant spectrum bands to transmit data. SUs also have their current residual energy and their location (x,y). A common control channel is assumed to be used among SUs to share information and to calculate the Euclidean distance to their one-hop neighbours.

The proposed clustering protocol consists of $r$ rounds and each round consists of two phases. First phase determines $\mathrm{CHs}$ and second phase optimally forms clusters to BS. The $\mathrm{CH}$ selection process combines both probabilistic model with FIS model. Each node in the network generates a random number between zero and one, then if this random number is less than the threshold value, the node becomes a candidate $\mathrm{CH}$ as described in equation (1).

The Fuzzy Inference System (FIS) is then used to calculate a chance parameter for each candidate CHs which define its possibility to become a $\mathrm{CH}$. After that, each $\mathrm{CH}$ advertises its chance value to neighbouring candidate $\mathrm{CHs}$ (Candidate_CH_Message). The CH which has the highest chance value in the neighbourhood will become a final CH and start cluster formation procedure. Other neighbouring candidate nodes who have lower chance values will reset their status to normal nodes. This strategy eliminates redundant $\mathrm{CHs}$ in the same vicinity. $\mathrm{CH}$ selection algorithm is presented in algorithm 1 . 
Vol. 8, Issue 2, February 2019

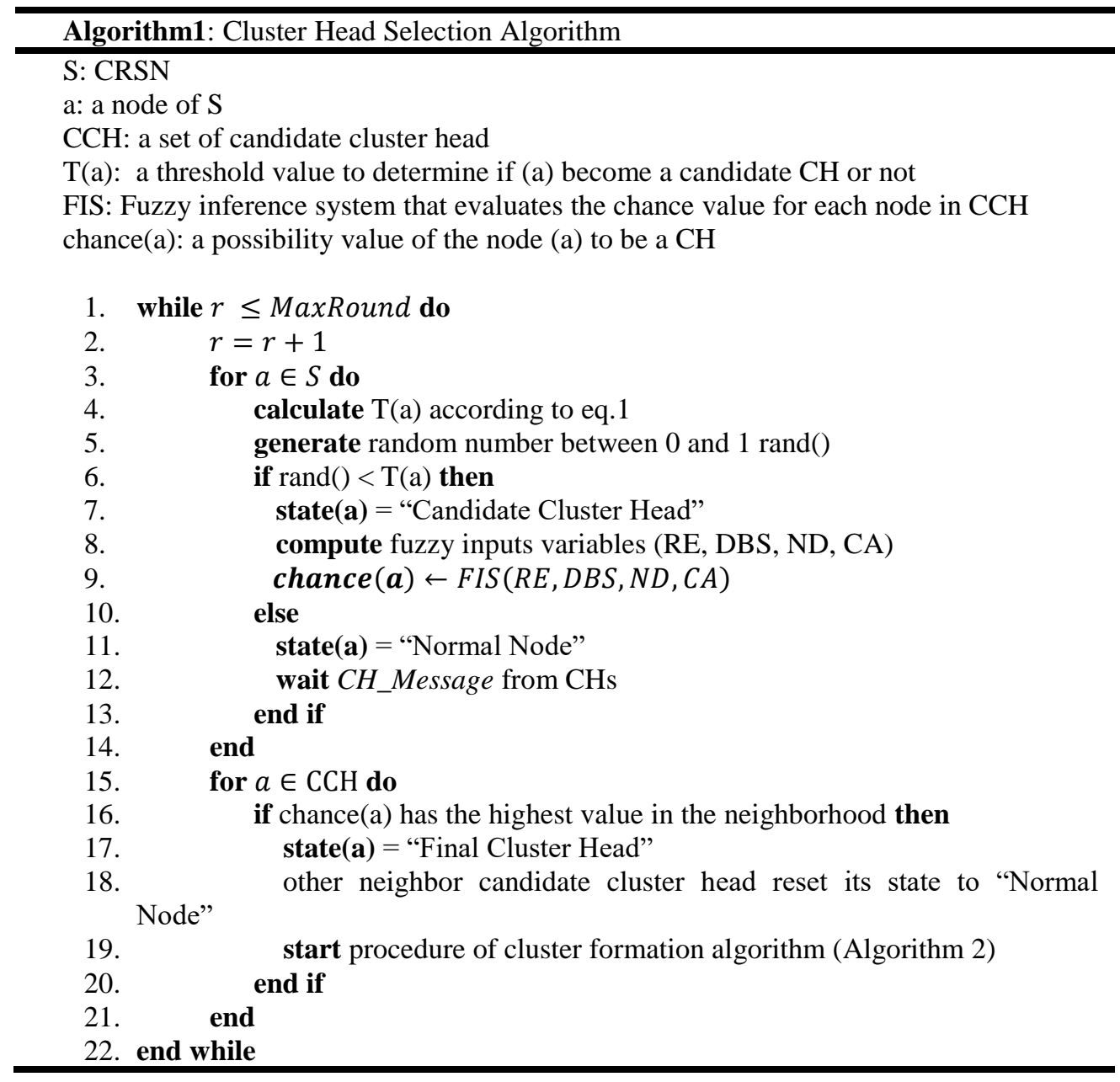

\section{A. The Proposed Fuzzy Inference System}

The FIS consists of four parts: fuzzification, knowledge base, aggregator, and defuzzification. In the proposed FIS model, for each candidate $\mathrm{CH}$, four descriptors are used as input parameters. These are defined as follows. First the residual energy (RE) which is the energy level available in each node and it is represented as the fuzzy variable RE. Second, the distance to BS (DBS) which is the distance between each node and BS and it is represented as the fuzzy variable DBS. Third the node degree (ND) which is the number of neighbour nodes within a specified radius $r$ and is it represented as the fuzzy variable ND, where radius is calculated as defined in equation (2).

$$
r=\sqrt{\frac{R}{\pi \times N \times P}}
$$

Where $\mathrm{R}$ is the area of the network (Length * width), $\mathrm{N}$ is the number of sensor nodes in the network, $\mathrm{P}$ is the desired percentage of $\mathrm{CH}$ in the network. Finally the channel availability (CA) is the ratio of the number of idle channels available to the node to the total number of channels. The CA(i) of node $\mathrm{i}$ is determined using equation (3).

$$
C A(i)=\frac{c_{i}}{m}
$$

Where, $m$ is the total number of channels (i.e. $|C|$ ) and $c \_i$ is the number of idle channels available to node $i$. The node with a higher value of channel availability means that the node has better opportunity to form a more stable cluster, which in turn decreases the energy consumption and increases the lifetime of the network due to avoiding unnecessary re-clustering. Thus this node will have a better chance to become $\mathrm{CH}$.

Each of these four input parameters is divided into three levels of linguistic values to reflect a different degree of membership of input linguistic variable. The four input descriptors are specified as follows: low, adequate, and high are 
used for RE variable. Close, medium, and far are used for DBS variable. Low, medium and high are used for ND variable. Finally low, medium and high are used for CA variable. The structure of the proposed FIS model is shown in Figure 1.

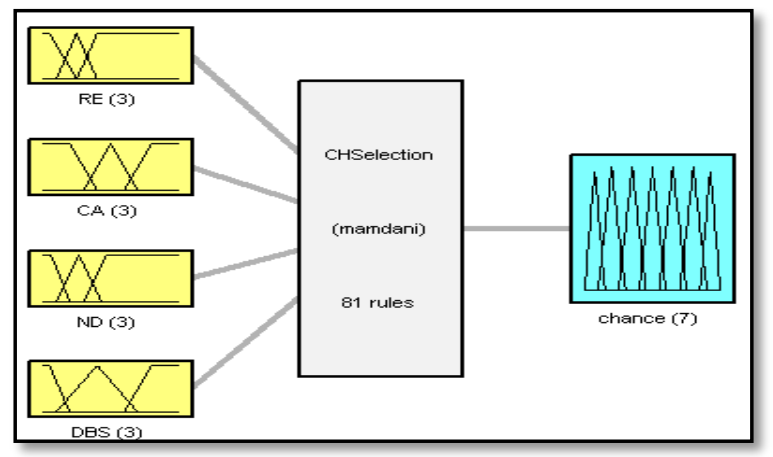

Fig.1 Fuzzy inference system for $\mathrm{CH}$ selection.

There are numerous types of membership functions such as Triangle, Trapezoidal, Sigmoidal, Gaussian, S-shape, and Z-shape. The triangle and trapezoidal membership functions are the most useful types due to their simplicity and easy of determining their degrees of membership. Therefore, the middle level of the four inputs (adequate, medium) is represented by triangle membership functions, while the other levels of linguistic values are represented by trapezoidal membership functions. The output linguistic variable which is the required chance have a seven linguistic values. These are very low, low, med low, med, med high, high, very high.

Finally after calculating the chance value, each candidate $\mathrm{CH}$ broadcast its chance which increases with RE, ND, and CA and decreases with DBS. Since there are four inputs parameters, each of them have three levels, therefore a total of 81 fuzzy IF-THEN rules are used. These rules fall between these two cases:

Case (1): If RE is low, DBS is far, ND is low, and CA is low then the chance is verylow.

Case (2): If RE is high, DBS is close, ND is high, and CA is high then the chance is veryhigh.

\section{B. Cluster Formation Algorithm}

The CHs with the highest chance value in its neighborhood will become a final CHs and start cluster's formation process. Clusters are formed by grouping neighbor nodes sharing common vacant channels. Each node senses the channels and maintains a channel status table that contains the PU appearance probability (p) and average PU idle time (T) for each channel. The PU appearance probability and PU idle time statistics are used to select the common data channel for a cluster.

A CH starts cluster formation process by determining channels that have lower $\mathrm{p}$, higher $\mathrm{T}$ and a higher number of onehop neighbor nodes. Channel $i$ with maximum weight $W_{i}$ defined in equation (4), is selected as the CDC for the cluster.

$$
\mathrm{W}_{\mathrm{i}}=\frac{1}{\overline{\mathrm{P}}_{\mathrm{i}}} \times \overline{\mathrm{T}_{\mathrm{i}}} \times\left|\mathrm{N}_{\mathrm{i}}\right|
$$

where, $\bar{P}_{i}$ is the average $p$ of the channel $i$ for all nodes in the cluster, $\bar{T}_{i}$ is the average $T$ of the channel $i$ for all nodes, and $\mathrm{N}_{\mathrm{i}}$ is the neighbour nodes of cluster head available on the channel $i$. The $\bar{P}_{i}$ and $\bar{T}_{i}$ of channel $i$ for a cluster head $A$ are calculated by the equations (5) and (6), respectively.

$$
\begin{gathered}
\bar{P}_{i}=\frac{\sum_{\forall k \in N_{i}} p_{i}(k)+p_{i}(A)}{\left|N_{i}\right|+1} \\
\bar{T}_{i}=\frac{\sum_{\forall k \in N_{i}} T_{i}(k)+T_{i}(A)}{\left|N_{i}\right|+1}
\end{gathered}
$$

After selecting the common data channel, the $\mathrm{CH}$ sends (CH_Message) to all neighbor nodes available on the selected channel and wait for the JOIN_REQ_Message from the nodes in N_i. The normal nodes receive (CH_Message) from CHs and join to the closest CH. Then normal nodes send JOIN_REQ_Message to the chosen CH. The cluster formation procedures is depicted in algorithm 2. 
Vol. 8, Issue 2, February 2019

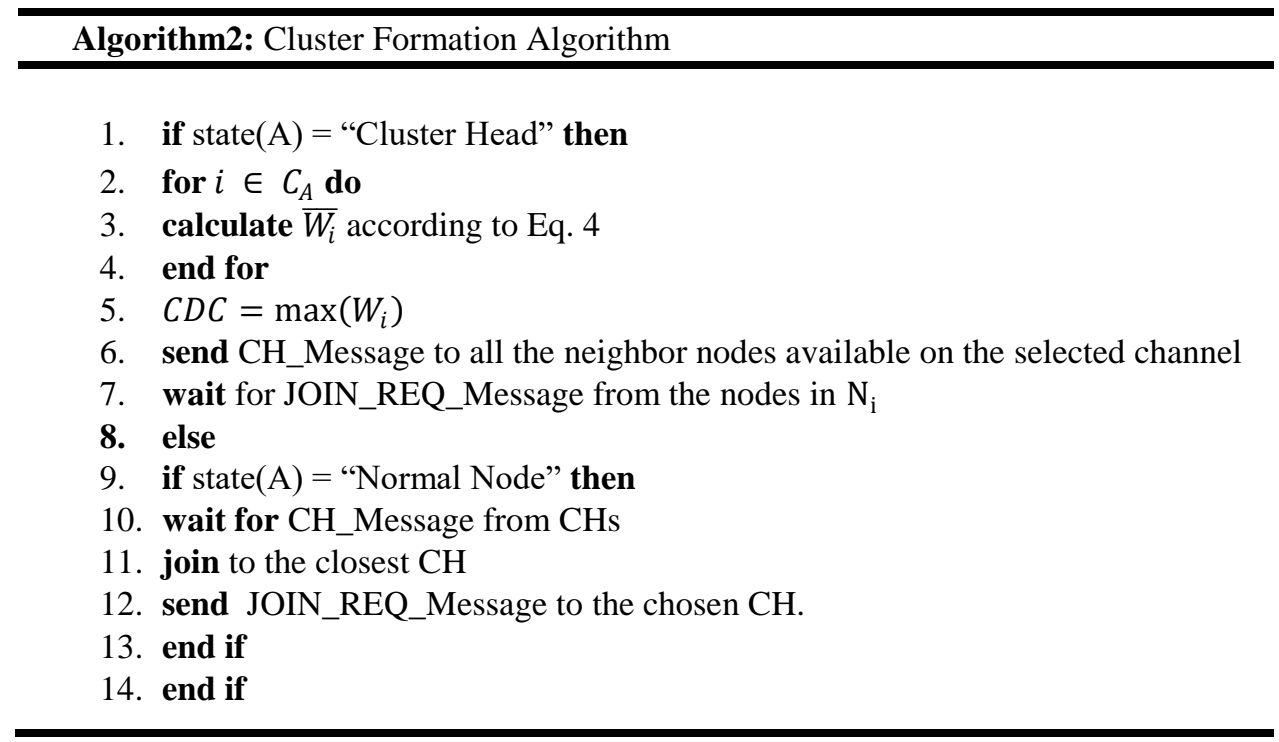

\section{III.PERFORMANCE EVALUATION AND RESULT ANALYSIS}

The performance of the proposed FEESA protocol is evaluated through extensive simulation in MATLAB. The proposed protocol is compared with three different protocols. The parameters used for performance evaluation are listed in Table I.

TABLE I SIMULATION PARAMETERS

\begin{tabular}{|c|c|}
\hline Parameter & Value \\
\hline Simulation rounds & 8000 rounds \\
\hline Area of Network & $100 \times 100 \mathrm{~m}^{2}$ \\
\hline Base Station Locations & $\begin{array}{l}\text { Centre of the field }(50,50) \\
\text { Outside the field }(50,175)\end{array}$ \\
\hline SU Nodes & $100,150,200,250$ \\
\hline PU Nodes & 10 \\
\hline Transmission Range & $20 \mathrm{~m}$ \\
\hline No. of Available Channels & 6 \\
\hline$P_{\text {free }}$ & 0.5 \\
\hline Packet Size & 4000 bits \\
\hline Initial Energy & $1 \mathrm{~J}$ \\
\hline Radio Electronics Energy $E_{\text {elec }}$ & 50 nJ / bit \\
\hline Amplifier energy $E_{a m p}$ & $100 \mathrm{Pj} / \mathrm{bit} / \mathrm{m}^{2}$ \\
\hline Data aggregation Energy $E_{D A}$ & $5 \mathrm{~nJ} /$ bit / signal \\
\hline
\end{tabular}


Vol. 8, Issue 2, February 2019

Our implemented ESAC protocol is not event-driven and it is called ESAC-x. ESAC-x protocol uses the same weighting criteria in $\mathrm{CH}$ selection: node degree, number of available channels and distance to sink. Both ESAC-x and CogLEACH are simulated as examples of spectrum-aware protocols, while LEACH protocol is simulated as a reference clustering protocol.

Two different simulation scenarios are used to evaluate the performance. The first scenario considers location of BS at the center of the network field, while the second scenario considers BS located outside the field of the network.

Three performance metrics are evaluated. The first is network lifetime which is the number of rounds till First Node Dead (FND). Second is energy consumption (measured in Joule) and represents rate at which energy is dissipated by sensor nodes in a WSN within a specific time. Third is the number of packets sent to BS which is the total number of packets sent by all sensor nodes in the network to the BS.

\section{A. Network Lifetime}

Fig.2 (a) shows the number of rounds at which the first node die out in the network for the four simulated protocols while varying number of nodes for the first case of positioning BS in the centre of network field. The number of rounds till FND in LEACH protocol ranging between 500 and 600, while for spectrum aware CogLEACH protocol ranging between 1700 and 2000 with improvement more than $200 \%$ over LEACH protocol results. The results of ESAC-X protocol ranging between 2400 and 2600 with improved more than $300 \%$ over LEACH protocol results and approximately $34 \%$ over CogLEACH protocol results. The results of the proposed FEESA protocol ranging between 2700 and 3200 with enhancement more than $400 \%$ over LEACH protocol results, and approximately $60 \%$ over CogLEACH results, and approximately $21 \%$ over ESAC-x results.

Figure 2 (b) shows the results when moving the position of base station outside the network field. The number of rounds at which FND for LEACH protocol ranging between 169 and 208, while for CogLEACH protocol this number is ranging between 518 and 610 with an improvement of approximately 200\% over the LEACH protocol results. The results of ESAC-x protocol ranging between 1205 and 1369 with an improvement of more than 600\% over LEACH protocol results and approximately $134 \%$ over $\mathrm{CogLEACH}$ protocol results. The results of the proposed FEESA protocol ranging between 1299 and 1680 with an improvment of more than 700\% over LEACH protocol results, and approximately $174 \%$ over CogLEACH results, and approximately $17 \%$ over ESAC-x results.

This scenario shows that the proposed FEESA protocol has the best results over other examined protocols when varying number of network nodes. This improvement in network lifetime is due to impact of proposed optimal selection strategy for $\mathrm{CHs}$ based on fuzzy logic that uses four different selection parameters of: distance to the BS, residual energy, node degree, and channel availability.

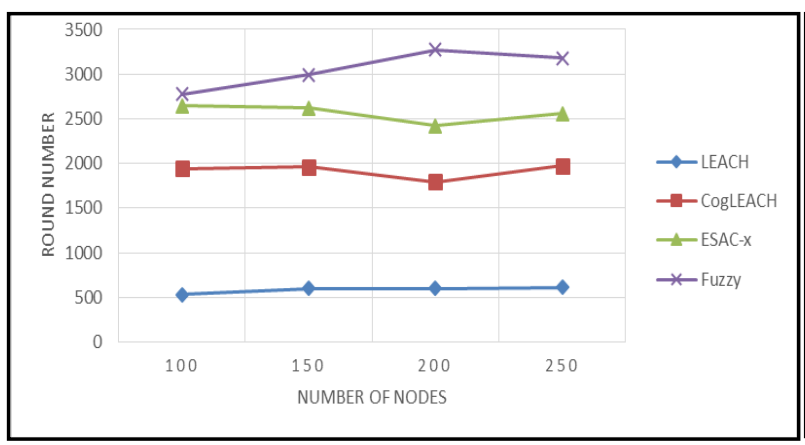

(a)

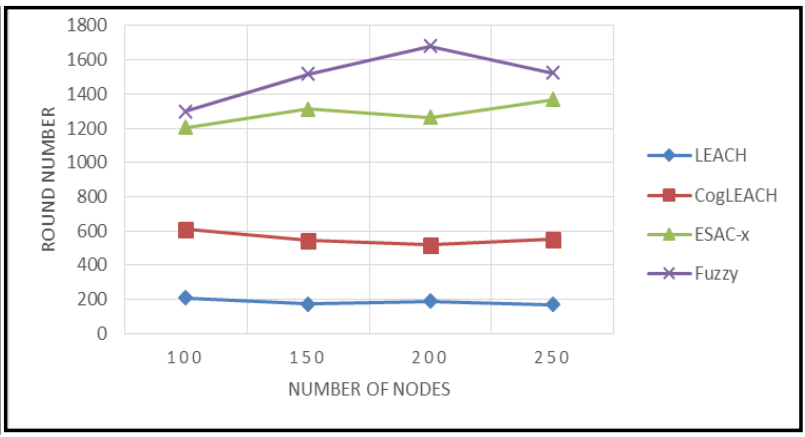

(b)

Fig.2 Network lifetime when varying number of network nodes (a) when the position of the BS is in the center of network field, (b) when position of BS outside the network field.

\section{B. Energy Consumption}

Fig.3 (a) shows the consumption of energy for four examined protocols when the position of BS is at the centre of the network field. LEACH protocol consumed the highest energy by approximately $97 \%$ in the middle of the simulation rounds and reaches $99 \%$ at the end of the simulation. The energy consumption of CogLEACH protocol in the middle of the simulation is approximately $75 \%$ and $92 \%$ at the end of the simulation. In ESAC-x, protocol this energy consumption approximately $71 \%$ in the middle of simulation and $87 \%$ at the end of the simulation. The proposed FEESA protocol has the lowest energy consumption by approximately $61 \%$ in the middle of the simulation rounds and reaches $78 \%$ at the end of the simulation. 
Vol. 8, Issue 2, February 2019

Fig.3 (b) shows the energy consumption for the four examined protocols when the position of BS is outside the network field. LEACH protocol gives the highest energy consumption by approximately $99 \%$ in the middle of the simulation rounds and reaches approximately $100 \%$ at the end of the simulation. The energy consumption of CogLEACH protocol in the middle of the simulation is approximately $96 \%$ and $97 \%$ at the end of the simulation. In ESAC-x protocol, the energy consumption approximately $95 \%$ in the middle of simulation and $97 \%$ at the end of the simulation. The proposed FEESA protocol still has the lowest energy consumption by approximately $82 \%$ in the middle of the simulation rounds and reaches $93 \%$ at the end of the simulation.

The proposed FEESA protocol shows the best results with respect to energy consumption over other examined protocols. This improvement is due to cluster formation strategy which maintains both energy efficiency and spectrum awareness. The transmission over the available vacant channel ensures the reliability of communication and decreases packet loss rate which decreases consumption of energy.

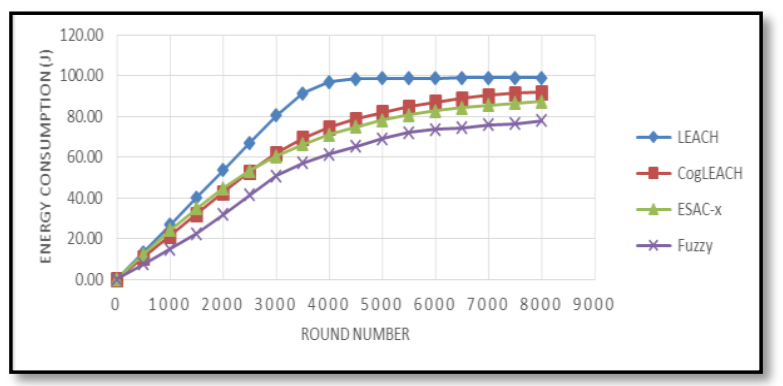

(a)

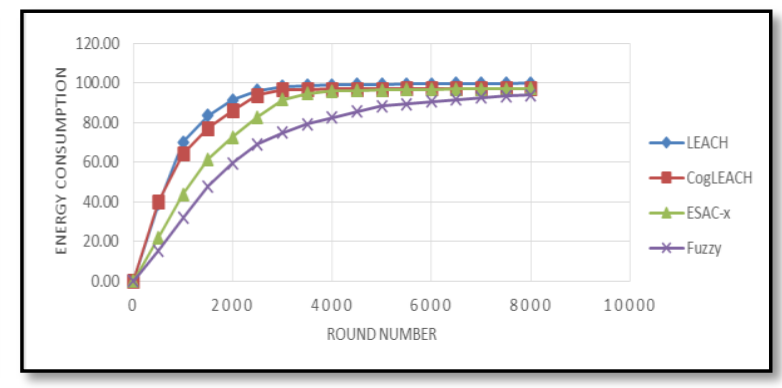

(b)

Fig.3 Total energy consumption when varying number of rounds (a) when the position of BS is in centre of the network field, (b) when the position of BS is outside the network field.

\section{Number of Packets sent to BS}

Fig.4 (a) shows number of packets sent to BS in each round when the position of BS is at the centre of the network field. LEACH protocol gives the highest number of packets sent to the BS. CogLEACH protocol gives the lowest number of packets. ESAC-x and FEESA protocols give approximate results with a slight advantage for proposed FEESA protocol.

Fig.4 (b) show the number of packets sent to BS in each round when the position of BS is outside of the network field. LEACH protocol gives the highest number of packets sent to the BS. CogLEACH protocol gives the lowest number of packets. ESAC-x and proposed protocols give approximate results with advantage for FEESA protocol.

In general, the LEACH protocol gives the best throughput over the other examined protocols due to the high percentage of nodes that are involved in sending the packets to BS more than other protocols that effect network lifetime and energy efficiency.

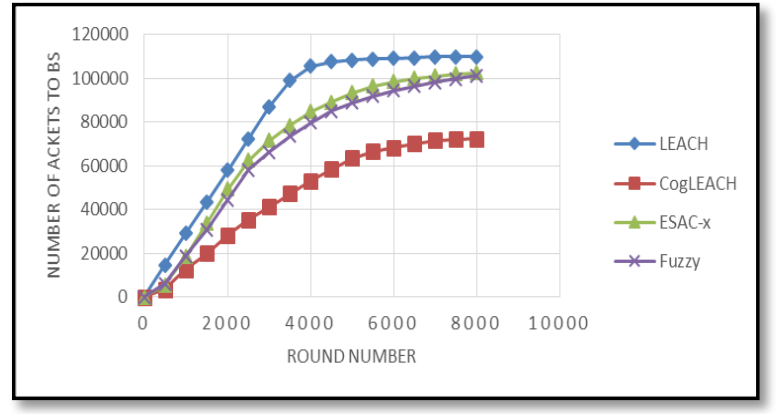

(a)

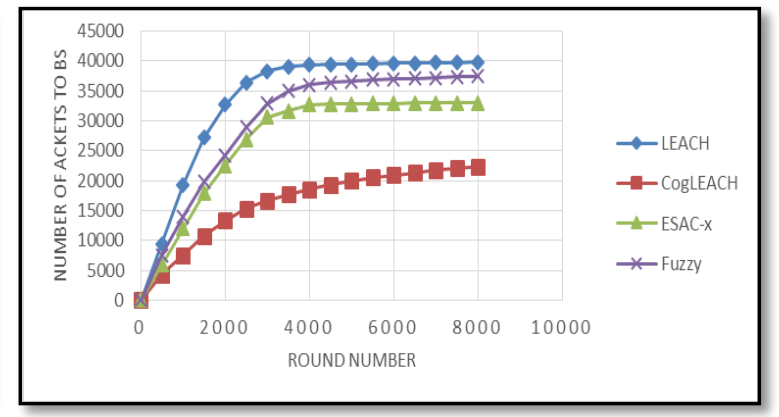

(a)

Fig.4 Number of packets sent to BS when varying number of rounds (a) when the position of BS is in the center of network field, (b) when the position of BS is outside the network field. 


\section{IV.CONCLUSION}

This paper developed an Energy Efficient and Spectrum Aware cluster-based routing protocol (FEESA) based on fuzzy logic to optimally elect CHs using four fuzzy parameters of distance to BS, remaining energy, node degree and spectrum availabilities. The simulation results showed the effectiveness of the proposed protocol in extending network lifetime and reducing energy consumption. The improvement is due to optimal selection criteria and the cluster formation strategy. However, this paper assumes that clustering takes place at the beginning of simulation, however a re-clustering algorithm needs to be developed to take into consideration PU's activities during simulation rounds.

\section{REFERENCES}

[1]. K. Sohraby, D. Minoli, and T. Znati, Wireless sensor networks: technology, protocols, and applications: John Wiley \& Sons, 2007.

[2]. B. Bhushan and G. Sahoo, "Routing Protocols in Wireless Sensor Networks," in Computational Intelligence in Sensor Networks, ed: Springer, 2019, pp. 215-248.

[3]. W. R. Heinzelman, A. Chandrakasan, and H. Balakrishnan, "Energy-efficient communication protocol for wireless microsensor networks," in System sciences, 2000. Proceedings of the 33rd annual Hawaii international conference on, 2000, p. 10 pp. vol. 2.

[4]. O. Younis and S. Fahmy, "HEED: a hybrid, energy-efficient, distributed clustering approach for ad hoc sensor networks," Mobile Computing, IEEE Transactions on, vol. 3, pp. 366-379, 2004.

[5]. M. Al Hadidi, J. S. Al-Azzeh, O. P. Tkalich, R. S. Odarchenko, S. O. Gnatyuk, and Y. Y. Khokhlachova, "ZigBee, Bluetooth and Wi-Fi Complex Wireless Networks Performance Increasing," 2017.

[6]. R. Biswas, "Spectrum Sensing Techniques: An Overview," in Sensing Techniques for Next Generation Cognitive Radio Networks, ed: IGI Global, 2019, pp. 125-132.

[7]. S. Haykin, "Cognitive radio: brain-empowered wireless communications," Selected Areas in Communications, IEEE Journal on, vol. 23, pp. 201-220, 2005.

[8]. O. B. Akan, O. B. Karli, and O. Ergul, "Cognitive radio sensor networks," Network, IEEE, vol. 23, pp. 34-40, 2009.

[9]. S. Zubair, N. Fisal, Y. S. Baguda, and K. Saleem, "Assessing routing strategies for cognitive radio sensor networks," Sensors, vol. 13, pp. 13005-13038, 2013.

[10]. R. M. Eletreby, H. M. Elsayed, and M. M. Khairy, "CogLEACH: A spectrum aware clustering protocol for cognitive radio sensor networks," in Cognitive Radio Oriented Wireless Networks and Communications (CROWNCOM), 2014 9th International Conference on, 2014, pp. 179-184.

[11]. A. Latiwesh, "Energy efficient spectrum aware clustering for cognitive sensor networks," Master Thesis, Electrical and Computer Engineering, Concordia University Montreal, Canada, 2016.

[12]. M. Ozger and O. B. Akan, "Event-Driven spectrum-aware clustering in cognitive radio sensor networks," in INFOCOM, 2013 Proceedings IEEE, 2013, pp. 1483-1491.

[13]. M. Ozger, E. Fadel, and O. Akan, "Event-to-sink Spectrum-Aware Clustering in Mobile Cognitive Radio Sensor Networks," 2015.

[14]. M. Tabassum, M. A. Razzaque, M. N. Miazi, M. Hassan, A. Alelaiwi, and A. Alamri, "An energy aware event-driven routing protocol for cognitive radio sensor networks," Wireless Networks, pp. 1-14, 2015/08/22 2015.

[15]. I. Gupta, D. Riordan, and S. Sampalli, "Cluster-head election using fuzzy logic for wireless sensor networks," in Communication Networks and Services Research Conference, 2005. Proceedings of the 3rd Annual, 2005, pp. 255-260.

[16]. A. K. Singh and N. Purohit, "An optimised fuzzy clustering for wireless sensor networks," International Journal of Electronics, vol. 101, pp. $1027-1041,2014$

[17]. J.-M. Kim, S.-H. Park, Y.-J. Han, and T.-M. Chung, "CHEF: cluster head election mechanism using fuzzy logic in wireless sensor networks," in Advanced communication technology, 2008. ICACT 2008. 10th international conference on, 2008, pp. 654-659.

[18]. J. Anno, L. Barolli, F. Xhafa, and A. Durresi, "A cluster head selection method for wireless sensor networks based on fuzzy logic," in TENCON 2007-2007 IEEE Region 10 Conference, 2007, pp. 1-4.

[19]. S. Gajjar, M. Sarkar, and K. Dasgupta, "Cluster head selection protocol using fuzzy logic for wireless sensor networks," International Journal of Computer Applications, vol. 97, 2014.

[20]. E. H. Mamdani and S. Assilian, "An experiment in linguistic synthesis with a fuzzy logic controller," International journal of man-machine studies, vol. 7, pp. 1-13, 1975 . 